\title{
Research Report on the Diversified Development Mode of Elder Support Industry in Yantai
}

\author{
Pingping Yang \\ Yantai Nanshan University \\ E-mail: 603483773@qq.com
}

\begin{abstract}
It is very necessary to construct a multi-level, multi-structure and multi-function elder support industry mode. In general, there are advantages and disadvantages in the development of the elder support industry in Yantai, so there are both challenges and opportunities. In the face of broad market prospects, there is regional development imbalance and imbalance of resources distribution. The development directions of elder support industry are as follows: the supply market develops in an initial stage and there is lack of market supply to a great degree. The elder support industry market develops towards subdivision and industrialization. The elder support industry has become a "sunrise industry". Quite a few domestic provinces and cities are increasing the support to elder support industry. The market supply for elder support industry has not yet formed the scale, and enterprises and merchants fail to seize this business opportunity.
\end{abstract}

Keywords-Yantai; the elder support industry mode; development direction

\section{THE DEVELOPMENT STATUS OF THE ELDER SUPPORT INDUSTRY IN YANTAI}

By the end of 2011, the city's total population over 60 has reached 1263400 people, accounting for $19.38 \%$ of the total population, with a nearly doubled increase compared with 1990. At the same time, the elderly population in Yantai is gradually showing the trend of empty nest. As of the end of 2011, there are a total of 557900 elderly households in Yantai. Among them, there are more than 320000 pure senior citizen families (empty nest families), accounting for $57.43 \%$. The statistical data in May 2013 show that among more than 6500000 people in Yantai, the population over 60 surpassed 1300000, ranking second in the whole province. In 2015, the aged population may increase to 1480000 , accounting for $22.7 \%$ of the total population. By 2020 it will reach 1810000 , accounting for $27.7 \%$ of the total population. There is averagely one senior citizen in every four people. The aged tendency of population is very serious.

In the face of the grim situation of aging population, the traditional family support and the elder support from the government and enterprises increasingly are difficult to take on the heavy burden of elder nursing services. The number of aged population in Yantai has entered into a period of rapid growth, and the aging problem is very serious. It is the priority of the whole society to treat senior citizens, help senior citizens spend their remaining days happily from the material aspect and get spiritual pleasure and happiness and solve the problem of supporting old people. Nearly 500000 empty nesters are potential customer base of elder support institutions. The existing elder support mode can't meet the needs of the market. According to incomplete statistics, there are 201 elder support institutions in Yantai with a total of 35000 beds for old people. In other words, there are only 27 beds for every thousand of old people averagely. It is hard to get one bed. People even need to wait for more than a year to get one bed. However, there are a few of people in ordinary elder support institutions due to their poor infrastructure.

The population size of old people in Yantai is large and the speed of growth is fast. It has become a problem that the society is concerned about to help the aged spend their remaining days, reduce and eliminate the negative effect of the aging of population. From the evolution of the elder support industry in Yantai, it is experiencing the transformation from traditional "home care" to socialized modern "community and home care" and other diversified mode.

\section{The Construction of Multi-Level, Multi- STRUCTURE AND MULTI-FUNCTION ELDER SUPPORT INDUSTRY MODE}

According to the distribution and physical conditions of the elderly in Yantai, the main demand of the elderly in Yantai is the "solitary" elder support service mode, the "semi-solitary" elder support service mode and the "gregarious" elder support service mode.

\section{A. The "solitary" elder support service mode}

The "solitary" elder support services are mainly targeted at the elderly who are physically better and able to live independently and care about the value of independent survival and the spirit of self-sufficiency, and not willing to leave the previous living environment. These old people have no self-care ability and their children have no time to take care of them. The "solitary" elder support service mode introduces the socialized elder support service mode on the basis of "home care". It provides housebound elderly with services, such as health maintenance, security and everyday life care.

The people aged from 60 to 70 are called as "the young old". Most of them have the self-care ability, so there is 
relatively less demands of social elder support among this group.

\section{B. The "semi-solitary" elder support service mode}

The "semi-solitary" elder support service mode is mainly for the elderly who are healthy but have no self-care ability. For example, they can walk independently but cannot cook. They live in elder support institution in the daytime and go home for sleep at night. It can solve the problem of lonely situation of the elderly whose children cannot take care of them during the day and enable the elderly stay together with their children. Therefore, this kind of elder support mode is very popular in foreign countries. This service mode generally is the old group over 65. From Monday to Friday, elder support institutions arrange special cars to receive and send the elderly, and provide them with breakfast, lunch, daily drinking water and fruits.

\section{The "gregarious" elder support service mode}

The "gregarious" elder support service mode refers to the boarding service elder support institutions. These institutions are for old people who cannot take care of themselves in daily life. The service institutions provide professional treatment and medical care. The cost of this elder support mode is relative high. Old people are completely separated with their children. Their children only visit them occasionally, so these old people are lack of emotional support. Therefore, these old people do not choose the elder support mode until they are really in need of it.

In the whole process of elder support, the daily care problem of the advanced ages is particularly prominent. The elderly over 80 are the group of people that mostly rely on daily care due to their healthy problems. They may have a lot of children, but it increases children's burden and they cannot receive professional care if they only rely on their children. Therefore, it is the best choice to hire professional nanny or move into the elder support institutions. Thus, the older the elderly are, the more possibly they may choose to select the social elder support mode.

According to the economic conditions of the elderly people in Yantai and the elder support awareness and the government's support policies, the elder support industry mode mainly includes "high-end" elder support service mode, "guarantee-type" elder support service mode and "povertytype" elder support service mode.

\section{D. "High-end" elder support service mode}

"High-end" elder support service mode is mainly for people who pay attention to the improvement of economic life, the satisfaction of materials life, and physical and mental health and the spiritual comfort. According to the economic development level of Yantai, they can provide perfect social security and medical service for the elderly. The direction of "high-end" elder support mode is to satisfy the material life and spiritual needs of the elderly.

"High-end" elder support service mode is characterized by: large scale, high level and sustainable development. The large scale means the large number. General nursing homes, apartments for the elderly and elderly care center cannot match with it in scale. It can meet the requirements for highlevel elder support functions. It is conducive to establishing scientific and reasonable proportional relationship among all kinds of industries and people, improving the utilization efficacy of colleges for the elderly, medical institutions, and thus forming a small town with elder support as theme. The high level means that the elder support service level is higher than the service level of relief elder support institutions and ordinary elder support institutions. It is mainly supported by: safe and reliable food, advanced and thoughtful medical care, advanced facilities, natural and harmonious interpersonal relationship, and high-quality environmental landscape. The sustainability means that to a certain extent it can dock the national macro intentions with the desire of private investors to make money, unify the construction of elder support project and the construction of farmer and modern agriculture of surrounding countryside, and connect the development of elder support facilities and equipment and modern service. Thus, we can guide the transformation of economic growth mode, effectively avoid short-term act and excessive speculation and protect the sustainable development of the system.

1)Travel vacation elder support mode. With good ecological environment, Yantai has the inborn advantage of developing elder support industry. Due to different landforms, climate conditions and natural factors, there are different advantages for elder support in different regions. The long-distance health maintenance and elder support refer to the elder support mode that the elderly leave their existing house and live in other places. The essence is to move to other places for health maintenance and elder support. There are many types of health maintenance and elder support, such as travel vacation and returning back to hometown. With the improvement of people's living standards, the elder support types have been transformed from home care elder support, community care, elder support institution to travel vacation and migrant elder support and other diversified types. They winter in the south and summer in the north. They travel around and enjoy their elder support life. Nowadays, the new long-distance elder support mode is gradually accepted by more and more old people.

Elderly people show special preference to travel. After they experienced their busy and tightening professional life, they hope to live their remaining days breezily, harmoniously and meaningfully. They travel famous mountains and rivers, and enjoy the joy of travel. It has become an important choice for many old people. The travel projects, such as "senior tourism", "sunset red" are popular among the elderly. Travel also gradually becomes one fashion many older people pursue. For the elderly group, the "sunset red" economic-type recuperation tourism products appear. The "sunset red" recuperation tourism products are low in pricing, large in transparent (only including accommodation) and flexible in arrangement. It gives more respect for the elderly, and is very suitable for the travel characteristics of slow pace and low intensity of old people, and so many old people prefer it. Travel agencies in Kunming, Haikou, Ji'nan, Chongqing and other places have 
put forward many targeted recuperation tourism products for the elderly, such as Hainan Recuperation Travel, aiming at the potential market.

Yantai's government calls for the integration of ecological tourism resources, medical resources, and health culture resources, to foster the elder support culture tourism industry with elder support as core, elongate elder support leisure travel industrial chain and promote the interactive development of elder support service, tourist attractions, hotel dining, entertainment and other industries. In Longkou Nanshan, Kunyu Mountain, Muping Longquan, Yantai Development Zone Cishan Hill and Qixia AI Mountain, the scenery is unique and beautiful, suitable for establishing vacation elder support base.

The Foguang Recuperation Valley project built by Longkou Nanshan Real Estate builds is trying to put elder support settlements projects. The project put the concepts of elder support and health maintenance covering four major systems: health maintenance, settlement, entertainment and pastoral experience. The project focuses on the construction of residence and community, and put a lot of energy in the construction of health care center, leisure recuperation center and other supporting facilities. Even they establish the senior citizen association, university for the elderly, art society for the elderly owners to enrich cultural life of the elderly.

2) The community service elder support mode. The community service elder support mode refers to the concentrated residence zone for the elderly that can provide housekeeping, medical treatment, social entertainment and other service, integrating life and elder support function together and fitting for psychological feature of the elderly. In the elder support residence, it is to carry out standardized management, scientific operations, and efficient service. It is currently the world's most popular elder support mode. The related industries involved in elder support residence projects include health services, home service, equipment, insurance, finance, real estate, tourism and entertainment, education, consulting services, and etc. for the elderly.

Real estate enterprises absorb the health maintenance concept and construct the diversified elder support and health maintenance platform integrating living, leisure, entertainment and health care together to strengthen the main development body of elder support industry. It is suggested to develop rehabilitation aids, articles of daily use, food and drug, clothing and walking aid, nursing, fitness and other elderly supplies and service products, guide shopping malls, supermarkets, and wholesale markets to set up area exclusive for the elderly, and form a complete elder support service industry chain integrating elder support service, medical rehabilitation, catering and trade, entertainment, travel and vacation and article manufacturing. Communities can provide housekeeping, transport, medical care and other door-to-door services, and it can enhance the activities of daily living and activity scope of the elderly at home and guarantee the elderly to have a satisfied life.

The most attractive place of communities is the largescale community recreation center and a complete outdoor fitness place. This mode fits for the elderly who are healthy and can live independently. The elderly are more independently than the elderly living in nursing home. The United States has formed a diversified elder support system, and the solitary mode is an ordinary elder support mode. The high-end, intermediate and low-end houses can respectively meet the demands of the elderly of different ages and levels.

Vanke is constructing "Huoyue Home for the Elderly" in Beijing Fangshan. It is one form of elder support real estate. Vice President of Vanke Group and General Manager of Beijing Vanke Mr. Mao Daqing said, "There is still great space for the development of real estate in the 12th Five-year Plan. But there will be a big change in residential running mode when the turning point of population arrives in the 14th Five-year Plan". For traditional real estate, once the operators sell houses, they lose primary income. But for elder support real estate, the operators shall maintain a series of daily business, such as medical care, housekeeping, health maintenance, and business entertainment after they sell houses. Though it may consume more energy and investment, it could bring long-term steady income. It is in favor of sustainable development of the company. And it is in line with the future development direction of real estate industry. Japan's real estate industry also attaches importance to the elder support housing market, and its products are mainly divided into two types: one is the leisure and health care housing, and the other is medical treatment housing. Some developers grasp this opportunity to develop elder support real estate for the elderly, or provide house modification service, or integrate this design into real estate project with elder support characteristics. Take community elder support as priority. They can develop elder support service items, such as diet and daily life, washing clothes, cooking, bathing and accompanying nurse, provide care for the old couple or single elderly who have daily life ability but have no children to take care of them. It is suggested to send the elderly who can not live alone and have no children to take care of them to nursing home, provide financial support to the elderly in the community, such as installing facilities freely, reducing or remitting taxes. In addition, give spiritual care to the elderly, develop psychological counseling, teach them knowledge of health maintenance, use social resources to improve life environment of the elderly and seek support from social resources. In British, the government invested money to construct communities with comprehensive service function for the elderly. The community activity centers are the social entertainment place of the elderly. The foreign elder support cause and industrial development are established on the economic conditions. The development of elder support mode is closed related to national conditions of a country. Though some modes cannot be directly introduced, they can still provide prospective enlightenments and reference.

There is a huge demand for the real estate market for the elderly. The Research Department of China's Aging Society believes that as of the end of 2005, the elderly with a considerable purchasing power are concentrated in the southeast coastal areas and some large and medium cities in China. In ten years, the huge purchasing power of elderly market will be fully revealed when the elderly of 50 years 
old arrive 60. Most of them are successful in career. Their retirement allowance and savings are highly improved compared with that of the elderly over 60 at nowadays. Their consumption concepts are also different. This group is the earliest parents with only one child. They may rely on community service and select a different elder support mode because they have fewer children. The so-called top-grade consumption will become ordinary consumption after ten years. Middle and high income groups of elderly people have a large demand for high-end apartments for the elderly. The accelerating flow of social population makes more old people lacking of children's care. In the development of aging population, the demand of the elderly for housing has gradually changed. In some big cities, the majority of parents are willing to live separately with their children. It makes activities of the elderly undisturbed and reduces the burden of their children and friction among them in the long term. The facilities in ordinary house cannot satisfy the physical and psychological demands of the elderly, so it has become the first choice for the elderly with high income to live in a town for the elderly with complete facilities and considerate service.

\section{E. “Guarantee-type" elder support service mode}

In rural areas, the land production material is in a dominant position. The rural areas are often more backward than the cities, and the farmers are relative lower in income. The traditional family support is still the main elder support mode. However, with the continuous improvement of the urbanization rate, people's income level and the social security system are further improved. More and more people tend to choose the social elder support.

Japanese old man can live a "one-stop" life. To move into the elderly villages is not a great burden to a Japanese old man from a middle income family. The elderly villages also provide door-to-door diagnosis and nursing service as long as the elderly make an appointment in guild hall. At the same time, Asian countries are influenced by the Confucian culture, especially the filial piety culture. The care for the elderly is regarded as a personal virtue. It is the duty of the children, so the family elder support mode is generally advocated and encouraged by the government through policies. With the development of the society, the elder support mode is developed from family to society. The combination of home care elder support and social elder support is currently the most popular elder support mode in Japan. Taking advantage of the large scale of medical care and nursing industry, it will develop into a new service industry to boost the development of future economy. The door-to-door nursing service for the elderly will be the focus in the future development.

Establish new towns with elder support as theme. This new urbanization is an inevitable path to the optimization of urban and rural structure, the transformation of economic growth mode and the promotion for the construction of modern agriculture. It is a grand measure to realize a well-off society in an all-round way. The small elder support-themed towns take full account of the landscape environment and farmers' demand in project area. It can promote the mutual coordination of new urbanization and agricultural modernization. In view of the demand of elder support industry for quiet environment, we should choose places with favorable climate, lush vegetation, moderate scale and relatively independent field with appropriate proportion of mountains, rivers and farmland, 40 minute's drive from the city. Considering basic conditions and industrial vitality, it can be an important part in the construction of urbanization.

\section{F. "Poverty-type" elder support service mode}

The so-called "poverty-type" elder support service mode refers to that the elderly use facilities and services provided by social elder support institutions freely or with little cost and spend their remaining life. Social elder support is an inevitable trend of social productive forces and social development. China's social elder support is divided into civil welfare elder support and tradable elder support. The former mainly includes civil welfare houses for the elderly without legal support, economic income and labor ability, and other special group. They are set up by civil welfare administrations. The latter refers to various tradable elder support service institutions facing the whole society, including community elder support institutions, nursing homes and seniors' home and apartment for the elderly at different levels.

The elderly groups with low income have a large demand of welfare elder support institutions. Influencing by big rural and urban and regional economic gap, the elderly consumption groups in economic backward areas are limited in consumption ability, and they still stay on the concept of home care elder support and rearing children for old age. In the short term it is difficult for the elderly to have higher demand on the apartments for the elderly. Their demands mainly are welfare elder support apartments.

Our current elder support industry has the characteristic of meager profit, which is closely related to the reality. It is feature in the initial stage of this special industry. On the one hand, our economy is not well developed, and the people's income level is not high, and let alone the income of the elderly. If it is operated completely according to market rules, most old people cannot afford to buy service products in the market. The purchasing power is poor and thus it is unable to attract private investment. It certainly affects the development of elder support industry. On the other hand, the government at all levels has taken a series of supporting policies to support this industry, and made requirements for enterprises on welfare. These two factors determine that enterprises shall run elder support business in a meager profit way.

The apartment for the elderly is the cornerstone of security needs of the elderly. The increasing of empty nest family and the change of family structure give serious challenges to elder support of poor families. It can not fully meet the security needs of the poor elderly. The selection of elder support mode is directly related to the conditions of living environment. Many new communities have not built supporting facilities for elder support service or do not leave space for elderly service. The old communities are difficult 
to be developed. The public elder support institutions are small in scale, and it is difficult for the poor elderly to enter in. The private elder support nursing homes are low in charge, but their infrastructures are not complete, and they lack professional personnel. The contradiction between supply and demand is serious. Therefore, the supporting policies are effective approaches to promote the private elder support institutions. The government should state that not all elder support institutions must be non-profitable. The concept of welfare should be understood in broad sense. As long as the institutions satisfy the elder support demand of the elderly, can the institutions perform the function of social welfare regardless of its profit condition. The policies should give different support to profitable and non-profitable institutions in order to promote the social financing environment development in elder support.

\section{CONCLUSION}

According to conditions of some elder support institutions in Yantai, it is very necessary to construct a multi-level, multi-structure and multi-function elder support industry mode. In general, there are advantages and disadvantages in the development of the elder support industry in Yantai, so there are both challenges and opportunities. In the face of broad market prospects, there is regional development imbalance and imbalance of resources distribution. The development directions of elder support industry are as follows: the supply market develops in an initial stage and there is lack of market supply to a great degree. The elder support industry market develops towards subdivision and industrialization. The elder support industry has become a "sunrise industry". Quite a few domestic provinces and cities are increasing the support to elder support industry. The market supply for elder support industry has not yet formed the scale, and enterprises and businesses fail to seize this business opportunity.

\section{REFERENCES}

[1] Fu Hua. Social Elder support Mode and its Service Facilities. Journal of Capital Normal University (Natural Science edition), 2000, 02: 7279.

[2] Gao Xiuyan, Wang Na. Analysis of "Diamond Mode" of the Development of the Community Home Care Industry - Taking Shenyang City as an Example. Socialist Research, 2007, 06: 106-109.

[3] Gao Xiuyan, Yong Hengwu. Views on Introduction of Competition Mechanism in Urban Community Home Care Elder support industry. Modern Finance and Economics, Tianjin University of Finance and Economics Journal, 2009, 02: 18-21.

[4] Gong Jingyi. Home Care Elder support - Community Elder support Service: in Line with China's National Conditions. Journal of Hohai University (Philisophy and Social Sciences edition), 2004, 04: 72-74. 